\title{
Political topic-communities and their framing practices in the Dutch Twittersphere
}

\author{
Maranke Wieringa \\ Utrecht Data School, Utrecht University, Netherlands, maranke@dataschool.nl \\ Daniela van Geenen \\ University of Applied Sciences Utrecht, Netherlands, daniela.vangeenen@hu.nl
}

Mirko Tobias Schäfer

Utrecht Data School, Utrecht University, Netherlands, m.t.schaefer@uu.nl

\section{Ludo Gorzeman}

Utrecht University, Netherlands, ludo@nomennesc.io

Published on 15 May 2018 | DOI: 10.14763/2018.2.793

\begin{abstract}
In light of the need for political plurality and informed debate this study questions information distribution and curation on Twitter. We contribute to the understanding of ideological homophily by exploring the notion of the 'echo chamber'. Using a sample of two weeks of Dutch Twitter data, we combine network analysis of retweet networks, with qualitative reading and categorisation of engagement with media content in tweets within political topic communities. We found that media references were predominantly framed in affirmative ways in relation to the referenced medium content. Our findings show that users consciously select media messages that correspond with the general sentiment within their topic community, or frame them accordingly. We see this as a willful 'echo chamber', or a 'repillarisation'.
\end{abstract}

Keywords: Echo chambers, Framing, Political homophily, Social filtering, Social media practices

\section{Article information}

Received: 12 Dec 2017 Reviewed: 08 Mar 2018 Published: 15 May 2018

Licence: Creative Commons Attribution 3.0 Germany

Competing interests: The author has declared that no competing interests exist that have influenced the text.

URL:

http://policyreview.info/articles/analysis/political-topic-communities-and-their-framing-practices-dutc h-twittersphere

Citation: Wieringa, M. \& van Geenen, D. \& Schäfer, M. T. \& Gorzeman, L. (2018). Political topiccommunities and their framing practices in the Dutch Twittersphere. Internet Policy Review, 7(2). https://doi.org/10.14763/2018.2.793

This paper is part of Networked publics, a special issue of Internet Policy Review guest-edited by William H. Dutton. 


\section{INTRODUCTION}

In the wake of Brexit, the election of Donald Trump, and the rise of nationalist populism in Europe, policy makers are concerned about the way social media affect public opinion. Traditional media and politicians alike quickly blamed social media for fuelling hate speech, disseminating misinformation, and being prone to the manipulation of audiences. Inherent to these claims is a technological determinism that neglects users' media practices as much as institutional aspects in transforming public information retrieval and democratic debate. This paper analyses users' media practices of selecting and disseminating media messages in political topic communities on Twitter. For this purpose, we investigated the Dutch Twittersphere, paying particular attention to ways in which media content such as diverse journalistic contributions taken from mainstream media and alternative outlets are framed. Looking at the media practices, our analysis reveals conscious choices made by users about which media messages to select and how to frame them for the intended audience in different political topic communities on Twitter. It contrasts claims of manipulated users, widely distributed 'fake news', and users locked into 'filter bubbles'.

Our corpus originally consisted of two weeks of Dutch Twitter data, more than 7.6 million tweets, gathered between 4 and 18 September 2016 (see also Van Geenen et al., 2016). Based on a social network analysis, we have identified two comparatively large politically interested topic communities. Our first topic community of interest can be described as 'centre-left' and is both the largest (approximately 40,000 accounts) and a highly heterogeneous community. Our second community of interest is the '(far-)right' community, consisting of roughly 10,000 accounts. We have found this latter community to show both high connectivity and strong peaks in centrality in its top central accounts. We argue these central accounts act as ideological hubs and feel confident in labeling this community as an 'echo chamber'.

To shed light on the social factor in the filtering and sharing of particular content we empirically explore users' (cross-)media practices and the ways of framing media references herein. Sampling media references from both the 'center-left' and '(far-)right' communities allowed us to analyse the particular ways in which this content is received, framed, and negotiated within the overall Dutch Twittersphere and within said 'echo chamber' of a politically interested topic community. Fitting with the ideological oppositional role of alternative media, we have found the '(far-)right' community to distribute a far higher percentage of alternative media compared to the 'center-left' community. Of particular interest is our finding that references to mainstream media outlets that are traditionally considered leftist are also heavily referenced within the '(far-)right' community. Close reading showed that these references tend to be renegotiated and framed within the community's ideological position. We thus propose a view of 'echo chambers' not as being isolated from media that do not share an ideological position, but instead as a cherry-picking and framing practice which draws from a highly diverse set of media outlets.

\section{SOCIAL FILTERING AND POLITICAL HOMOPHILY}

Willson (2014) notes that social filtering has always taken place. In fact, in the Netherlands, this was common practice during the 'pillarisation', one of the examples referred to by Zuiderveen Borgesius et al. (2016) in demonstrating the "prevalence of self-selected personalisation" of 
media content through the ages. One could even say that the societal pillars in the Netherlands constituted 'filter bubbles' long before the advent of electronic media. With the term 'social filtering', Willson refers to a "selective engagement with people, communication and other information as a result of the recommendation of others" (ibid, p. 218). Yet, she points out that with the emergence of social media platforms the "politics of social framing" have changed and have to be reevaluated (ibid.). Drawing from Hermida (2012), Bruns identifies 'social sharing' as a distinct media practice in news dissemination (Bruns, 2018, p. 311). This practice, he continues, is a further unbundling of editorial platforms. Important for our case here is that users in topic communities pick from a wide range of available media (newspapers, radio and $\mathrm{TV}$, but also weblogs and social media pages) to select what they find 'newsworthy' for their followers.

However, recent research in media practices based on representative surveys of consumers of (online) media content in diverse countries indicates that the consumer behaviour of the larger part of these populations is characterised by cross(-platform) media practices (Dutton, Reisdorf, Dubois, \& Blank, 2017; Dubois \& Blank, 2018; Newman, Fletcher, Kalogeropoulos, Levy, \& Kleis Nielsen, 2017). Dubois and Blank (2018) emphasise "political interest" as a decisive moderating factor in the selection, and also self-selected personalisation - probably even polarisation - of internet users' (daily) media digest. Political interest, in general was defined as an indicator for identifying users who tend to avoid so-called 'echo chamber' (ibid.). In this study we are particularly interested in the ways in which such users select or filter and (subsequently) frame (cross-)media content on Twitter. Twitter is a comparatively open platform, allowing for interaction between various - also in terms of follow relationships - unrelated users; and it stimulates the (re)distribution of media content in the form of hyperlinks (e.g., Bruns \& Moe, 2014).

Thus, while the distribution of content based on assumed homophily is an undeniable aspect of social media platforms, it does not account for the actual media plurality and cross-media aspects of everyday media consumption. It puts an emphasis on selective media use as being constituted through social media, while newspapers, TV and radio stations have been catering to (politically) different audiences and, with the popularity of satellite TV in the 1980s, to fragmented audiences. Willson's notion of social filtering on online platforms describes essential aspects of opinion forming (Katz \& Lazarsfeld, 1955). In this paper, we specifically focus on social filtering as it applies to information dispersal on Twitter amongst politically interested topic communities, and their engagement with that media content.

Messages might include political narratives, memes, tropes, or other messages that are circulated within fringe communities and spread to other media platforms: Benkler et al. (2017) showed how the far-right blogosphere is informing mainstream media through mediators such as Breitbart and Fox News. Studying how mainstream and alternative media news from Reddit and 4Chan spread to Twitter, Zannettou et al. (2017) demonstrate that alt-right communities can influence dissemination of fringe content to mainstream social media platforms. These publications (Benkler et al., 2017; Zannettou et al., 2017) and our study demonstrate that topic communities, and even fringe communities are intertwined with mainstream media through social media and other mediators. In the case of political topic communities in the Netherlands, we noticed that new media platforms such as the provocative and populist weblog GeenStijl, the right-wing oriented The Post Online, and others were also intertwined with mainstream media platforms through ownership relations. GeenStijl is owned by the Telegraaf Group which also owns the Netherlands leading tabloid, and the media group Veronica Association is a majority 
shareholder of The Post Online.

Political discussion and ideological homophily on Twitter has been studied before by, for instance, Conover et al. (2011), who like Feller et al. (2011) found that groups with different political ideologies tend to be separated from each other. Conover et al. (2011) based their research on retweet behaviour and mentions in the period leading up to the congressional midterm elections in the US. They found that while the retweet structure of the network shows segregation, the mention network did paint a different picture: the segregated groups were found to have communicated with each other using mentions. Feller et al. (2011) instead opted for tracking those users who mentioned the German parties in light of the Bundestag elections. Using retweet and mentions/replies as measures, Boutet, Kim and Yoneki (2012) found that politically interested Twitter users were highly segregated as well during the 2010 UK general election. Barbéra et al. (2015), found that information tends to be circulated among people with a similar ideological stance, which is congruent with Colleoni et al.'s findings (2014). Barbera et al. (2015) also report that - in the American context - liberals were more likely to engage in what they term 'cross-ideological' debate. Along a similar train of thought, Boutyline and Willer (2015) found that conservatives displayed more homophily than liberals and moderates.

What our research contributes, is that we do not infer political preference from an account's following behaviour, but rather extract their preference from their everyday activity on Twitter. We extracted those two politically interested clusters from the entirety of ordinary Dutch Twitter activity within the timeframe of two weeks, having first created a retweet network of the complete Dutch Twittersphere. As we were interested in mundane Twitter activity, our research is placed outside of an election context, as opposed to many of the earlier studies on political communication. Moreover, we offer new insights in how politically interested communities engage with media content, such as news, opinion pieces and so forth, derived from traditional media, as well as other diverse media outlets.

\section{A NOTE ON 'ALTERNATIVE MEDIA'}

Our sample consists of tweets with links which reference mainstream media, such as newspapers, TV, and radio, but also new platforms such as Facebook and YouTube. Additionally, it contains references to what we want to address as alternative media. The term was originally coined to describe counterculture and critical media productions (Downing, 1984; Atton 2001; Couldry \& Curran, 2003; Fuchs, 2010) that describe visions different from, and render it possible to scrutinise, hegemonic narratives. For example, our samples reference blogs and other website publishing positions that are profoundly different from the consensus in mainstream media. While this observation needs a more elaborate examination in future research, from what we saw through the close reading of framing practices in tweets, accounts in the right wing cluster tend to refer to such alternative media more often than accounts in the other two clusters.

Next to content-related distinctions between mainstream and alternative media, another difference between the two kinds of outlets is between authorship and ownership. The editors and authors of many of the right-wing alternative media, mostly Wordpress blogs, hide behind pseudonyms; additionally, the owner of the domain remains anonymous.2 Ownership, however, is not a clear indicator for alternative media. In fact, some of the bigger alternative media such as Joop.nl, The Post Online and GeenStijl are owned by established mainstream media companies. Moreover, we noticed that new formats in terms of alternative media often disregard 
established journalistic codes of conduct, and lack clear genre indications. Yet, this observation deserves more in-depth research, also inquiring into the idea that these media outlets could cater to 'fake news'.

In this investigation we apply the term 'alternative media' in a comprehensive way. For us, as a working definition, it denotes all kinds of journalistic platforms which either voice a countercultural commentary, neglect a clear differentiation between various journalistic genres, do not uphold traditional journalistic codes of conduct, or do not make authorship and/or ownership explicit, as well as form a combination of these criteria. We want to distinguish between the aforementioned alternative media, and new journalistic venues which still operate within the scope of the traditional journalistic code of conduct. As new formats, we describe novel Dutch journalistic platforms such as De Correspondent, or Follow The Money.

\section{DATA SAMPLING AND METHODOLOGY}

The methodological strength of our investigation is formed by a combination of quantitative and qualitative approaches. Digital methods (Rogers, 2013) were employed to sample a data set based on language detection using a list of distinctive Dutch words (see Van Geenen et al., 2016 for more detailed information on the corpus collection). A Dutch sample constitutes a fruitful contribution to Twitter research as there are approximately 2.8 million registered Twitter accounts (Newcom, 2016) - compared to a population of 17 million people - and, according to our preliminary findings, we were able to identify almost 640,000 weekly active users (Van Geenen et al., 2016). These figures show a relatively high penetration of Twitter. For the collection of samples used for this investigation, we made an effort to choose a random timeframe on Twitter, unaffected by particular societal events, and, as much as possible, related topics of debate.3

Since we focus on (re)distribution practices in the Dutch Twittersphere, our starting point in detecting relevant clusters are retweet practices (e.g., Bruns and Moe, 2014), and the ways in which they connect users on the platform. Earlier research indicated that retweets (RTs) are frequently used in affirmative modes, approving either the media content, or the user who shared this content, or both (e.g., Paßmann, Boeschoten, \& Schäfer, 2014; Paßmann, 2018:164). Therefore, in our effort to identify like-minded networked publics RT practices served as a vantage point. From more than 7.6 million tweets, we distilled nearly 2.2 million RTs. Based on this sample we performed social network analysis by mapping interactions (Bastian, Heyman, \& Jacomy, 2009) and computationally detected communities using the Louvain method for community detection in large networks (Blondel, Guillaume, Lambiotte, \& Lefebvre, 2008). The latter method seeks to identify groups of nodes (users) in the network that have more edges (retweets) within their particular group than outward edges (retweets of users outside of their group).

For the communities that were detected in this manner, we have calculated a number of metrics: the cluster's modularity and density, and the PageRank (Page et al., 1999) of the nodes within the clusters. Modularity refers to the ratio between the number of inward edges and the total number of edges originating from within the cluster. Moreover, communities with a high modularity generally distribute content from within their own community and show relatively weak links to the rest of the network (e.g., Newman, 2006). Density refers to the ratio between the number of edges inside a cluster and the total possible amount of edges for a cluster with that amount of nodes. A cluster in which every node has an edge to every other node will have a 
density of 1 ; more tight-knit communities generally have higher density, and lower density indicates a loose overall coherence. The PageRank score of a particular node within a cluster indicates the centrality and 'importance' of that node within the cluster. Nodes with higher PageRanks are likely to enjoy more exposure throughout the entire cluster; nodes with lower PageRanks are likely to reside in the outer rim of the cluster, enjoying relatively little exposure within the cluster. The classification of nodes with very low PageRanks as part of a particular community tends to be more ambiguous and prone to error than that of nodes with high PageRanks.

We combined the former 'distant reading' strategies (Moretti, 2013) with interpretative tactics of close reading of accounts and the media content they shared, supported by participatory observations of the Dutch Twittersphere. For the purpose of studying media references in relation to the public debate, we focused on two clusters in said RT network. The first cluster concerns the largest cluster in terms of users connected through their RT behaviour. It consists of more than 40,000 nodes representing unique Twitter accounts. While this cluster features, amongst others, accounts that can be identified as representing a centre-left political position based on their profile information (albeit a very heterogeneous one), the second selected cluster predominantly features accounts that are sympathetic to (far-) right political ideas, and shows stronger homogeneity. This cluster is constituted by approximately 10,000 nodes.

Subsequently, we departed from the accounts in both clusters to search for references to traditional and online-only media. In this step we decided to include not just RTs, but also all other kinds of tweets in order to inventory the diverse kinds and layers of framing applied by users. In this course, we found almost 387,000 article links distributed by accounts in the larger, centre-left cluster, and 345,785 links shared by accounts in the right-wing cluster, compared to more than 2.8 million media references in the total retweet network (which amounts to approximately $14 \%$ and $12 \%$ of all links). The selection is deliberately not limited to traditional media, such as (websites of) newspapers, TV or radio stations, but also includes online-only media, such as blogs, new journalistic platforms or alternative media outlets.

To avoid skewness in the sample selection we decided to filter out messages (re)distributed by accounts hosted by leading (traditional) media outlets such as accounts of the Dutch daily newspapers De Telegraaf and NRC. References to web content from both newspapers are prominently represented in the total sample of tweets that contain media references. Moreover, we prepared weighted random samples of the tweets that included media references for qualitative analysis. Weight was given to the PageRank score (Page et al., 1999) of a node within a particular cluster, indicating the centrality of the node within the cluster. This introduces a bias away from the ambiguous outer rim of the cluster where it is often unclear whether a node really belongs to the assigned cluster or may just as well be part of the neighbouring cluster. Instead, we focused our sampling on the central nodes within the cluster which more clearly belong to their respective communities. These samples are derived from both selected clusters, as well as from the entire network in order to establish a baseline sample, which resulted in three test samples. Each of the samples contains 500 tweets that are qualitatively assessed based on their modes of framing the media references.

In order to close-read the tweet content and explore the layers of framing herein, we employed Stuart Hall's (1973) model of encoding/decoding to understand how Twitter users disseminate mainstream and alternative media messages. Hall proposed a model of three possible decoding positions: dominant-hegemonic - what we term 'affirmative' - negotiated, and oppositional. Ross (2011) proposed to add another dimension to Hall's model. Whereas Hall focused on the reception side, Ross proposed to take the sender's encoding strategy into account as well. Ross' 
adaptation resulted in a scheme in which encoding/decoding strategies can, but do not need to overlap. Implicitly, we adapt Ross' (2011) take on Hall's (1973) model, by adding a third party. There are not just two parties involved when we discuss the uptake and dissemination of information on social media. Instead, there are, in fact, three: the media, the framer, and the audience (Wieringa, 2017).

We have analysed the frames by close-reading a random sample of messages, and the links embedded in them. In order to adequately categorise the framer's encoding strategy as either affirmative, negotiated, or oppositional (see figure 1 for an overview of the categorisation), we took the medium (message) as a baseline, in order to measure the secondary reaction. Within the negotiated tweets, we further categorised in which way the tweet was framed (e.g., emotive, illustration for broader debate). Aside from the manner of framing, we also categorised the subject of the frame (medium in its entirety/medium content).

\begin{tabular}{|c|c|c|}
\hline \multirow{12}{*}{ 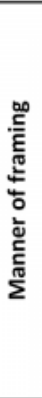 } & \multicolumn{2}{|l|}{$\begin{array}{l}\text { Affirmative } \\
\text { Endorses the (message of the) medium. }\end{array}$} \\
\hline & \multicolumn{2}{|l|}{ Negotiated } \\
\hline & \multicolumn{2}{|c|}{ Semi-endorses the (message of the) medium, but for instance places different accents. Comes in different forms: } \\
\hline & Positive emotion & Positive emotion is expressed (e.g. joy). \\
\hline & Negative emotion & Negative emotion is expressed (e.g. sadness/anger). \\
\hline & Change of tone & Text is charged with a different tone (e.g. sarcasm). \\
\hline & Twisting argument & Argument is bent so that it supports a different case. \\
\hline & Judgement & A judgement is expressed (e.g. "what a bad article"). \\
\hline & Article used to illustrate topic or debate & The content is used an example of a broader phenomenon. \\
\hline & Part of content highlighted & Only a portion of the content is used, the rest is ignored. \\
\hline & \multicolumn{2}{|l|}{ Oppositional } \\
\hline & \multicolumn{2}{|c|}{ Actively disputes the (message of the) medium) } \\
\hline
\end{tabular}

\begin{tabular}{|c|c|}
\hline \multirow{2}{*}{ 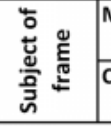 } & $\begin{array}{|ll|}\text { Medium } & \\
& \text { Subject is the medium in its entirety. }\end{array}$ \\
\hline & $\begin{array}{ll}\text { Content } & \\
& \text { Subject is only a particular message. }\end{array}$ \\
\hline
\end{tabular}

\begin{tabular}{|c|c|}
\hline 完 & $\begin{array}{l}\text { Degree of automation } \\
\qquad \text { Measure of automation in the tweet (e.g. news bots, or accounts linking to Facebook/YouTube updates) }\end{array}$ \\
\hline
\end{tabular}

Fig. 1. Categorisation scheme (building on Ross, 2011; Wieringa, 2017)

The initial operationalisation of Hall's/Ross' notions (Wieringa, 2017) has subsequently been adopted in commissioned research projects like those for the Dutch Association of Editors-inchief (Wieringa \& De Winkel, in press) and Vrij Nederland and Nieuwsuur (Broer \& Ostendorf, 2018). The method was found to produce similar results across different samples. The close reading was executed by two researchers intimately familiar with the coding scheme. Yet, as the close-reading was formulated as rendering exploratory insights, the samples of interest in this investigation were not doubly coded separately by the researchers.

\section{FINDINGS}

Our network analysis of media practices indicates topic-centred publics. In other words, we can discern clusters of users who converse about the same topics, and who share a particular viewpoint, or lifestyle. These topic-centred audiences often inherently 'crossmedia-reference' other media (print newspapers, other websites, radio programmes and frequently national TV, etc.) through their Twitter activity. These media references might also support beliefs and points of view of the specific topic-centred network. Looking at clusters of politically interested users provided us with insights concerning their media use, how they read and frame mainstream media messages and to what extent they reference other media, social media platforms, new journalistic formats, and alternative media. 
Our operationalisation of Hall's revised model of en/decoding showed that the majority of tweets examined through close reading were framed in affirmative ways in relation to the referenced medium content (between $60 \%$ and $70 \%$ in all three samples, see fig 2.). Moreover, the great majority of tweets framed the content instead of commenting on the entire medium. Thus, on the surface, it seems, that the most important thing is still what is said, not who is saying it.

\section{Manner of framing and subject of frame}

\begin{tabular}{|c|c|c|c|c|c|c|}
\hline Sample & 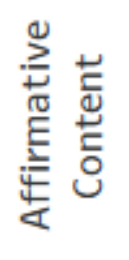 & 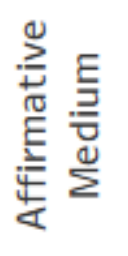 & 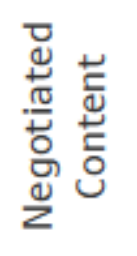 & 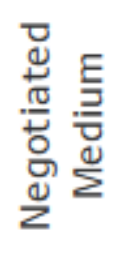 & 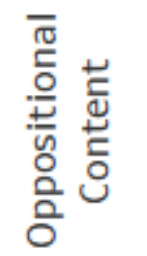 &  \\
\hline (Far-)right & 351 & 1 & 103 & 1 & 20 & 2 \\
\hline Base & 342 & 2 & 112 & 2 & 5 & \\
\hline Centre-left & 311 & 1 & 138 & 5 & 11 & \\
\hline
\end{tabular}

Amount

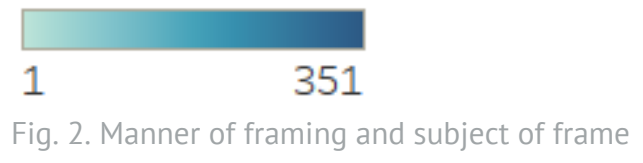

Yet, when we dig a little deeper and take into account which media outlets are the most dominant in the two clusters (and the entire graph), we see that this optimistic first conclusion needs severe nuancing. In the centre-left cluster the most dominant domains are those of mainstream media (nos.nl), (quality) newspapers as well as tabloids (volkskrant.nl, telegraaf.nl, nrc.nl), social media (youtube.com), and the mainstream online-only news website nu.nl. In the (far-)right cluster, we see a surprising amount of references to alternative media in the top six domains featured quite prominently as the second and third most referred to domains: tpo.nl, geenstijl.nl (online-only). Other top media references in this cluster are telegraaf.nl, ad.nl, and volkskrant.nl (tabloid and newspapers), nos.nl (mainstream media). Across the entire graph we see that social media (youtube.com, facebook.com), tabloids (telegraaf.nl, hln.be), sport-related news (sportinnederland.com), and mainstream media (nos.nl) are the most frequented (see figure 3 for a visual depiction). Interestingly, we see a Belgian tabloid in this list, which has to do with the Flemish 'bycatch' of our sampling, as we selected tweets based on language parameters. To sum up, while each cluster predominantly opts for an affirmative engagement with media content, we also see that these clusters use different media sources. The media sources which do overlap are prioritised differently.

In our qualitative exploration, the close-reading of tweets, we observed that the various clusters engage in different ways with the overlapping media. For instance, if medium a is present in group $b$ and $\mathrm{c}, \mathrm{b}$ and $\mathrm{c}$ will generally draw on different content. In other words, we see that when the centre-left refers to the tabloid Telegraaf or the (far-)right refers to the left-liberal Volkskrant, they predominantly refer to opinion pieces, or use a single article (separate from the rest of the newspaper) to make a particular point. This selective choice is of course supported by 
the media technology itself which affords to disseminate single articles taken out of context of the overall editorial line of the newspaper, and hence contributes to the unbundling of editorial products. It is not surprising that op-ed articles are more frequently selected for dissemination than so called long-reads or informative articles as they might correspond with the opinion or emotions of the user who distributes the (re-encoded) message.

Fig. 3. Distribution of top six linked domain names across clusters/entire graph.

\begin{tabular}{|l|l|l|l|}
\hline & Base & Centre-left & (Far-)right \\
\hline 1 & youtube.com & nos.nl & telegraaf.nl \\
\hline 2 & facebook.com & volkskrant.nl & tpo.nl \\
\hline 3 & telegraaf.nl & youtube.com & geenstijl.nl \\
\hline 4 & hln.be & telegraaf.nl & nos.nl \\
\hline 5 & sportinnederland.nl & nrc.nl & ad.nl \\
\hline 6 & nos.nl & nu.nl & volkskrant.nl \\
\hline
\end{tabular}

There seems to be a kind of disconnect between the two clusters. However, equating it to a 'filter bubble' (Pariser, 2011) is problematic, as the separate clusters do read/watch overlapping media. The phenomenon seems more like a willful 'echo chamber' in which the users amplify their own beliefs by focussing on those bits of information which support their worldview (resulting in a high amount of affirmative content frames), or select messages which they can negotiate in such a way that supports them. Alternatively, we could describe this as an ideological 'repillarisation' in the online environment, as people cluster together more strongly on the basis of ideology and select their information accordingly.

Boutyline and Willer (2015) mention "homophilous ties as building blocks of echo chambers" and introduce a method for measuring to which degree clusters of users constitute an echo chamber by tracing shared follower-relations to particular so-called ideological hubs (e.g., major politicians, journalists, etc.). Homophily to a particular ideological hub is measured as the ratio between the amount of followers of a particular user who share the same follower-relation to the ideological hub and the total number of followers of the particular user, leading to a score that describes to which degree the user's followers also follow the same hub. The higher the average homophily among all nodes within a cluster towards the set of leading ideological hubs within that cluster, the more this cluster can be regarded as an echo chamber. In our case, we can identify the major ideological hubs within the two clusters by examining the nodes with the highest PageRank within the cluster. Our analysis in terms of retweet-relations rather than follower-relations still gives good (or perhaps even better) insight in the overall exposure of tweets by these ideological hubs.

The top three PageRanks of our two clusters reveal a far steeper curve in the (far-)right cluster than in the centre-left cluster, going from 0.01713 to 0.00850 to 0.00754 in the (far-)right cluster compared to going from 0.00425 to 0.00366 to 0.00339 in the centre-left cluster. Furthermore, the top three of the (far-)right cluster is comprised of famous national politicians and columnists, whereas the top three of the centre-left cluster is comprised of a local politician, an anonymous account linked to a political movement, and a physician. As such, the (far-)right cluster shows much stronger signs of being centred around ideological hubs. Furthermore, comparing the modularity of the two clusters shows that the (far-)right cluster has a 
considerably higher ratio of inward versus outward edges (0.70) than the centre-left cluster (0.59). Finally, the density of the (far-)right cluster, a metric indicating the internal cohesion of the cluster, is nearly nine times higher than that of the centre-left cluster (0.00237 versus 0.00027). This tight-knit nature of the (far-)right cluster, as expressed by the high density, in combination with its high modularity and the steepness of the top PageRank leads us to conclude that there is a high degree of average homophily towards a small number of ideological hubs within the (far-)right cluster, the typical characteristic of an echo chamber. The same cannot be said for the centre-left cluster, lacking both clear ideological hubs in the centre of the network and the internal density to ensure exposure of these ideological hubs throughout the whole cluster.

This 'echo chamber' phenomenon is further supported by the overlap in popular hashtags in the clusters. This hashtag inventorisation showed that the politically interested topic communities do discuss similar topics (see figure 4 for a visual depiction). For instance, the television shows Pauw (\#pauw) and Zomergasten (\#zg16) were discussed both in the centre-left and the (far)right clusters, but were often discussed differently by those groups. On the other hand, we also see that the clusters have their respective focus areas. For instance the centre-left discusses healthcare and privacy, whereas the (far-)right often discusses the Party for Freedom (\#pvv) and the city of Zaandam, in which a handful of (Dutch) vloggers of Turkish descent were trolling their neighbourhood duringour sampling period - which sparked significant upheaval.

Fig. 4. Distribution of top six hashtags across clusters/entire graph.

\begin{tabular}{|l|l|l|l|}
\hline & Base & Centre-left & (Far-)right \\
\hline 1 & \#nieuwstwitter & \#zg16 & \#pauw \\
\hline 2 & \#nieuws & \#pauw & \#nieuws \\
\hline 3 & \#vacature & \#nieuwstwitter & \#pvv \\
\hline 4 & \#pauw & \#zorg & \#nieuwstwitter \\
\hline 5 & \#voetbal & \#privacy & \#zaandam \\
\hline 6 & \#actueel & \#buitenhof & \#zg16 \\
\hline
\end{tabular}

In terms of negotiating the disseminated medium content, the encoded tweets of accounts that sympathise with distinctive political positions indicated notable framing practices, particularly with regards to emotion and the way in which content is used to illustrate a larger debate. We found that the manners of negotiating medium content in both clusters are emotionally charged. Notably, the sentiment of negotiated reading is more negative in the far-right cluster than in centre-left cluster (figure. 5). Moreover, we found that the former group also utilises the content to illustrate a broader topic or debate more often (almost 40\%) than the latter (around 25\%). This observation poses the question in which ways manners of framing strain and distort media content, and consequently, the information conveyed. 


\section{Negotiation scatterplot: emotion and usage of message as illustration for topic/debate}

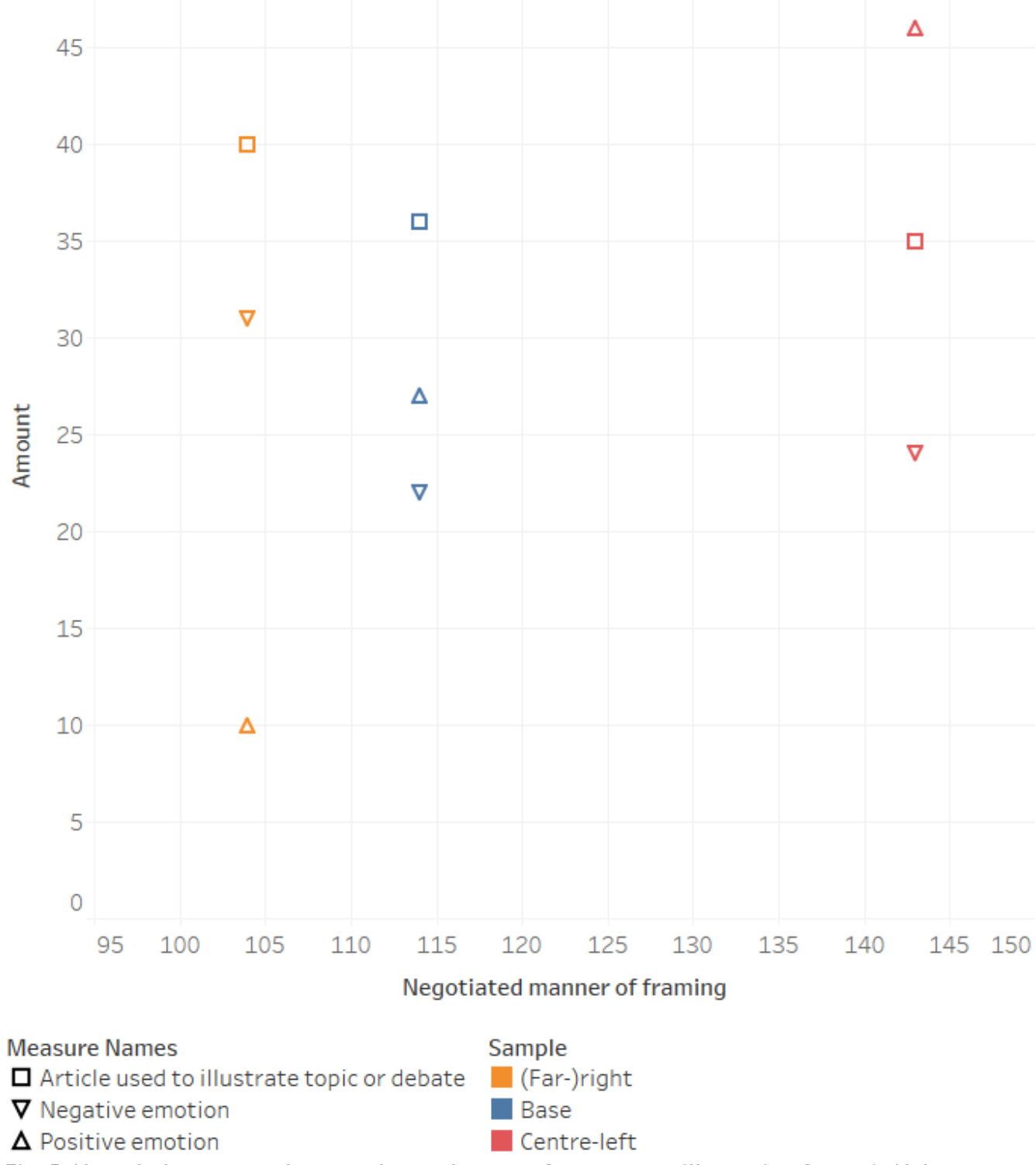

Fig. 5. Negotiation scatterplot: emotion and usage of message as illustration for topic/debate

Interestingly, we found few tweets which opposed (the) media (content). When we did find such oppositional comments, they were predominantly concerned with the media content, not the medium as a whole. This again underlines our observation that these politically interested topic communities heavily selected what they do (not) post online/retweet/engage with.

\section{CONCLUSION}

Our research provides information about how users actually engage with media messages and provides the groundwork for further in-depth analysis of media practices in a connected public sphere. In doing so, we contribute to an emerging debate on news curation through user dissemination and comments (Bruns, 2018). Politically interested Twitter users select from a rather broad range of different news sources, mainstream and alternative media. Selecting news 
items and disseminating them within their own topic community already constitutes a manner of framing, as the user places the selected item within the specific context of the common worldview. Often, an explanatory negotiation is not even necessary. This allows users in fringe communities such as the far-right to disseminate content even from politically opposed sources. The audience within the topic community is likely to read the content within the commonly shared worldview. We also note that the Dutch fringe communities most often reference the national tabloid which seems to represent news in a way that fits the shared worldviews. The alternative media are shared significantly less.

Our empirical work shows that news from the mainstream media in fact permeates deeply in both leftist and right-wing clusters - so it is not the dreaded (techno-deterministic) filter bubble we should fear, it seems. Our findings indicate that Twitter users predominantly use content which underlines their own views, standpoints, and so forth. In other words, the challenge that these (cross-)media practices pose is not that the media do not reach particular audiences; it is that these audiences cherry pick the content which suit them best and disregard the rest. This phenomenon, we argue, can be seen as a kind of willful 'echo chamber', or even a 'repillarisation'.

In societal terms, the findings we present could inform policy makers to review their perspective on so-called echo-chambers and misinformation online. Our findings counter the notion of 'manipulated audiences' and emphasise users' conscious choice in shaping their conversations through careful selection of messages to disseminate. Here the users emulate a practice campaigning politicians use in their own communication strategy, selecting those messages that are 'decoded' appropriately by the intended audience (Wieringa \& De Winkel, forthcoming).

It also might inform editors to review how their editorial products are used after publishing and how the different entities that make up the entire publication are circulated independently in the various topic communities. In view of the alternative media maintaining less strict journalistic codes of conduct, further research should inquire the economy of these media platforms, their reach and reception. Both politicians and journalists should question to what extent they actually want to amplify the sentiments voiced on these platforms and that are employed to mobilise parts of the electorate that are open for populism.

Moreover, as researchers we have to inquire, borrowing from Habermas (1989), to what extent a 'structural transformation of the public sphere' is unfolding through these media practices and the changing media landscape, and how this transformation is affecting the open society. 


\section{REFERENCES}

Atton, C. (2001). Alternative media. London, New Delhi: SAGE Publications.

Barberá, P., Jost, J.T., Nagler, J., Tucker, J.A., \& Bonneau, A. (2015). Tweeting From Left to Right: Is Online Political Communication More Than an Echo Chamber?. Psychological Science, 26(10), 1531-1542. doi:10.1177/0956797615594620

Bastian, M., Heymann, S., \& Jacomy, M. (2009). Gephi: An Open Source Software for Exploring and Manipulating Networks. In Proceedings of the Third International ICWSM Conference. AAAI Publications. Retrieved from https://gephi.org/publications/gephi-bastian-febo9.pdf

Benkler, Y., Faris, R., Roberts, H. \& Zuckerman, E. (2017, March 3). Study: Breitbart-led rightwing media ecosystem altered broader media agenda. Columbia Journalism Review. Retrieved from https://www.cjr.org/analysis/breitbart-media-trump-harvard-study.php

Blondel, V. D., Guillaume, J.-L., Lambiotte, R. and Lefebvre, E. (2008). Fast unfolding of communities in large networks. Journal of Statistical Mechanics: Theory and Experiment, 2008(10), 1-12. doi:10.1088/1742-5468/2008/10/P10008

Boutyline, A., \& Willer, R. (2016). The Social Structure of Political Echo Chambers: Variation in Ideological Homophily in Online Networks. Political Psychology, 38(3), 551-569.

doi:10.1111/pops.12337.

boyd, d. (2010). Social Network Sites as Networked Publics: Affordances, Dynamics, and Implications. In Z. Papacharissi (Ed.), Networked Self: Identity, Community, and Culture on Social Network Sites (pp. 39-58). New York: Routledge.

Broer, T. \& Ostendorf, C. (January 24, 2018). Hoe de boze twitteraar het debat bepaalt. Vrij Nederland. Retrieved from https://www.vn.nl/hoe-de-boze-twitteraar-het-debat-bepaalt/

Bruns, A. (2018). Gatewatching and news curation: Journalism, social media, and the public sphere. New York: Peter Lang.

Bruns, A., \& Moe, H. (2014). Structural Layers of Communication on Twitter. In K. Weller, A. Bruns, J. Burgess, M. Mahrt, \& C. Puschmann (Eds.), Twitter and Society (pp. 15-28). New York: Peter Lang.

Colleoni, E., Rozza, A., \& Arvidsson, A. (2014). Echo Chamber or Public Sphere? Predicting Political Orientation and Measuring Political Homophily in Twitter Using Big Data. Journal of Communication, 64(2), 317-332. doi:10.1111/jcom.12084.

Conover, M. D., Ratkiewicz, J., Francisco, M., Goncalves, B., Menczer, F., \& Flammini, A. (2011). Political Polarization on Twitter. In Proceedings of the Fifth International AAAI Conference on Weblogs and Social Media (pp. 89-96). AAAI Publications. Retrieved from https://www.aaai.org/ocs/index.php/ICWSM/ICWSM11/paper/view/2847

Couldry, N., \& Curran, J. (2003). Contesting media power: Alternative media in a networked world. Lanham: Rowman \& Littlefield Publishers.

Downing, J. (1984). Radical media: The political experience of alternative communication. New York: South End Press. 
Dubois, E. \& Blank, G. (2018). The echo chamber is overstated: the moderating effect of political interest and diverse media. Information, Communication \& Society, 21(5), 729-745.

doi:10.1080/1369118X.2018.1428656

Dutton, W. H., Reisdorf, B., Dubois, E., \& Blank, G. (2017). Search and Politics: The Uses and Impacts of Search in Britain, France, Germany, Italy, Poland, Spain, and the United States (Working Paper No. 5-1-17). Quello Center. Retrieved from https://ssrn.com/abstract=2960697

Feller, A., Kuhnert, M., Sprenger, T., \& Welpe, I. (2011). Divided They Tweet: The Network Structure of Political Microbloggers and Discussion Topics. In Proceedings of the Fifth International AAAI Conference on Weblogs and Social Media (pp. 474-77). AAAI Publications. Retrieved from

http://www.aaai.org/ocs/index.php/ICWSM/ICWSM11/paper/viewFile/2759/3210.

Fuchs, C. (2010). Alternative media as Critical Media. In European Journal of Social Theory, 13(2), 173-192. doi:10.1177/1368431010362294

Geenen, D. van, Schäfer, M. T., Boeschoten, T., Hekman, E., Bakker, P. \& Moons, J. (2016, October 5-8). Mining One Week of Twitter. Mapping Networked Publics in the Dutch Twittersphere. Paper presented at AoIR 2016: The 17th Annual Conference of the Association of Internet Researchers. Berlin, Germany: AoIR. Retrieved from

https://spir.aoir.org/index.php/spir/article/view/1394

Habermas, J. (1989). The Structural Transformation of the Public Sphere. Cambridge, MA: MIT Press.

Hall, S. (1973). Encoding and Decoding in the Television Discourse. Birmingham, England: Centre for Cultural Studies, University of Birmingham.

Katz, E., \& Lazarsfeld, P. F. (1955). Personal Influence: the Part Played by People in the Flow of Mass Communications. New York: The Free Press.

Moretti, F. (2013). Distant Reading. London: Verso.

Newcom (2016). Nationale Social Media Onderzoek 2016. Retrieved from http://www.newcom.nl/uploads/images/Publicaties/Newcom-Nationale-Social-Media-Onderz oek-2016.pdf.

Newman, M.E.J. (2006). Modularity and community structure in networks. Proceedings of the National Academy of Sciences 103(23), 8577-8582. doi:10.1073/pnas.0601602103

Newman, N., Fletcher, R., Kalogeropoulos, A., Levy, D., \& Kleis Nielsen, R. (2017). Reuters Digital News Report 2017. Oxford: Reuters Institute for the Study of Journalism. Retrieved from http:// www.digitalnewsreport.org/

Page, L., Brin, S., Motwani, R., \& Winograd, Terry. (1999). The PageRank Citation Ranking: Bringing Order to the Web. (Technical Report No. 1999-66). Retrieved from http://ilpubs.stanford.edu:809o/422/

Pariser, E. (2011). The Filter Bubble: What the Internet Is Hiding from You. New York: Penguin Press. 
Paßmann, J., Boeschoten, T., \& Schäfer, M. T. (2014). The Gift of the Gab: Retweet Cartels and Gift Economies on Twitter. In K. Weller, A. Bruns, J. Burgess, M. Mahrt, \& C. Puschmann (Eds.) Twitter and Society. New York: Peter Lang.

Paßmann, J. (2018). Die soziale Logik des Likes: Eine Twitter-Ethnografie. Frankfurt; New York: Campus.

Picard, R. G. (2000). Audience fragmentation and structural limits on media innovation and diversity. In J.J. van Cuilenburg \& R. van der Wurff (Eds.), Media and open societies: Cultural, economic and policy foundations for media openness and diversity in East and West (pp. 180191). Amsterdam: Het Spinhuis.

Rogers, R. (2013). Digital Methods. Cambridge, MA: The MIT Press.

Ross, S. (2011). The encoding/decoding model revisited. In International Communication Association Conference. Boston. http://urn.kb.se/resolve?urn=urn:nbn:se:su:diva-7146o

Wieringa, M. A. (2017). 'F' is for $f \square a \square k \square e \square \square n \square e \square w \square s / f \square i \square l \square t \square e \square r \square$ $\square b \square u \square b \square b \square l \square e \square$ s / framing: How politicians frame media (content) on Facebook \& Twitter during the Dutch Tweede Kamer elections of 2017 (Masters thesis). Utrecht University. Retrieved from https://dspace.library.uu.nl/handle/1874/352810

Wieringa, M.A. \& Winkel, T. de. (in press). The politician as editor-in-chief: News distribution on social media by candidates for the 2017 election for the Dutch House of Representatives. Media and Communication.

Willson, M. (2014). The politics of social filtering. Convergence, 2O(2), 218-232. doi:10.1177/1354856513479761

Zannettou, S., Caulfield, T., De Cristofaro, E., Kourtellis, N., Leontiadis, I., Sirivianos, M., Stringhini, G. \& Blackburn, J. (2017). The Web Centipede: Understanding How Web Communities Influence Each Other Through the Lens of Mainstream and Alternative News Sources. ArXiv:1705.06947 [Cs]. Retrieved from http://arxiv.org/abs/1705.06947

Zuiderveen Borgesius, F.J., Trilling, D., Möller, J., Bodó, B., Vreese, D.H. de, Helberger, N. (2016). Should we worry about filter bubbles?. Internet Policy Review, 5(1).

doi:10.14763/2016.1.401

\section{FOOTNOTES}

1. Pillarisation (Dutch: verzuiling) refers to the segregation of a society on the basis of religion or ideologies. Each ideology/religion corresponds to a societal 'pillar' which is comprised of media institutions, educational institutions, political parties, and so forth. The verzuiling in the Netherlands lasted from the late 1800 s to the mid/late 1900 .

2. Yet, of course such pseudonyms are not necessarily a signifier of the (far-)right, and may help to strive for free speech in countries where this is not entrenched in the constitution. Our argument in this matter, however, is specific to the Dutch context, in which such right to free speech is firmly entrenched in the constitution. Our line of thinking here draws from the German context, in which all websites are required to list the owner of the website by name.

3. Close reading of tweets revealed commotion, especially in the (far-)right cluster, concerning proposed legislation that would make donor registration the default option. This topic 
dominated the debate in the political topic communities during our two-week period. 